CTP-TAMU-96/91

hepth@xxx/9205035

\title{
Sine-Gordon Solitons as Heterotic Fivebranes
}

\author{
HoSeong La* \\ Center for Theoretical Physics \\ Texas A\&M University \\ College Station, TX 77843-4242, USA
}

\begin{abstract}
The Euclidean analogues of the sine-Gordon solitons are used as sources of the heterotic fivebrane solutions in the ten-dimensional heterotic string theory. Some properties of these soliton solutions are discussed. These solitons in principle can appear as string-like objects in 4-dimensional space-time after proper compactifications.
\end{abstract}

$3 / 92$

* e-mail address: hsla@phys.tamu.edu, hsla@tamphys.bitnet 
The study of solitons has been long pursued in various aspects of physics. It involves the investigation of nonlinear evolution equations in general. Now string theory is not an exception anymore. Lately the structures of the classical solitonic solutions of string theory have been actively investigated[1]. In particular, the heterotic fivebrane solution conjectured by Duff[2] and constructed by Strominger[3] exceptionally interesting in the sense that it is dual to the fundamental string in a generalized sense of the electric-magnetic duality 1 . However, most of the solutions known so far are rather ten-dimensional solutions so that their fate in 4-d space-time after compactification is still elusive.

Thus it is important to address a question that what would be the implications of the physics of the fivebrane in ten-dimension on the physics in four-dimensional space-time after some proper compactification. There may be some physical consequences due to the above duality, although such a duality does not necessarily require the existence of the dual object. For example, the origin of the electric-magnetic duality in four-dimension might be such a string-fivebrane duality in ten-dimension. In other words, the monopole solution in four-dimension might be related to the fivebrane solution in ten-dimension. This aspect was already advocated by Harvey and Liu [5]. The dynamical similarities between these two systems are investigated classically in ref. [6]. Furthermore, we could conjecture that the solitonic sector in four-dimension could be originated from the solitonic sector of ten-dimension.

In this letter, as a first step toward such structures in the solitonic sector of string theory, we attempt to investigate how these solutions appear in the lower dimensional subspaces of the $(1+9)$-dimensional space-time. By proper dimensional reductions imposing the Killing symmetries we make the fields in these subspaces independent from the rest of the space.2

Also in this letter we shall in particular examine whether sources other than YangMills (YM) instantons can provide fivebranes. We find that this is indeed possible. As an example we explicitly work out the case of the (Euclidean) sine-Gordon solitons. As is well known, the Euclidean sine-Gordon system does not allow any finite-action static solutions, namely instantons. But there are infinite-action static solutions, which are good enough for our purpose.

1 This duality which interchanges Noether charge (e.g. electric charge) and topological charge (e.g. monopole charge) is in principle the foundation for the Montonen-Olive conjecture[ [], which is yet to be confirmed rigorously.

2 Note that the Killing reduction does not change the dimensionality of the objects. 
Solitonic solutions of other systems reduced from the SDYM equation also can be used to construct fivebranes. To name a few more examples, the hyperbolic monopoles and vortices are among them. The detail will be presented elsewhere [7]. Also we can attempt to analyze the motion of strings in the fivebrane geometry inside $(1+3)$-dimensional spacetime, using the metric suggested here 8 .

\section{Heterotic Fivebranes}

A fivebrane is a five dimensional extended object and the existence of such a higher dimensional object is in some sense surprising. Nevertheless, such a solution exists in string theory. Let us first review the derivations given in refs. [3] [9]. The heterotic fivebrane is a solution to the equations of the supersymmetric vacuum for the heterotic string

$$
\begin{gathered}
\delta \psi_{M}=\left(\nabla_{M}-\frac{1}{4} H_{M A B} \Gamma^{A B}\right) \epsilon=0 \\
\delta \lambda=\left(\Gamma^{A} \partial_{A} \phi+\frac{1}{6} H_{A M C} \Gamma^{A B C}\right) \epsilon=0, \\
\delta \chi=F_{A B} \Gamma^{A B} \epsilon=0
\end{gathered}
$$

where $\psi_{M}, \lambda$ and $\chi$ are the gravitino, dilatino and gaugino, while the anomaly equation is given by

$$
\mathrm{d} H=\alpha^{\prime}\left(\operatorname{tr} R \wedge R-\frac{1}{30} \operatorname{Tr} F \wedge F\right)+\mathcal{O}\left(\alpha^{\prime 2}\right) .
$$

In the above we have properly rescaled all the field variables and that the string coupling $g_{s}=\mathrm{e}^{-\phi}$ and $\alpha^{\prime}$ are the only independent couplings. In the heterotic string theory $\alpha^{\prime}$ is proportional to $\kappa^{2} / g_{\mathrm{YM}}$, where $\kappa$ is the gravitational coupling constant.

The corresponding low-energy effective action for the heterotic string up to the leading order of $\alpha^{\prime}$ is

$$
\mathcal{S}=\frac{1}{\kappa^{2}} \int \mathrm{d}^{10} x \sqrt{-g} \mathrm{e}^{2 \phi}\left(R+4 \partial_{\mu} \phi \partial^{\mu} \phi-\frac{1}{3} H^{2}-\frac{1}{30} \alpha^{\prime} \operatorname{Tr} F^{2}+\cdots\right)
$$

where the dots include the fermionic part of the action that are not relevant for our purpose now.

The basic idea to have fivebranes is due to the observation that the transverse space to these extended objects is a four dimensional space, which is conformally equivalent to the Euclidean space, and they should appear as usual particle-like objects in such a transverse space. Thus it is convenient to work on the decomposed space $M^{1,9} \rightarrow M^{1,5} \times M^{4}$. Note 
that for solitons the Poincaré symmetry is not necessarily required, which makes such a decomposition possible.

In $(1+9)$-dimension we have Majorana-Weyl fermions, which decompose down to chiral spinors according to $\mathrm{SO}(1,9) \supset \mathrm{SO}(1,5) \otimes \mathrm{SO}(4)$ for such a decomposition. For such spinors the dilatino equation eq.(2) is satisfied by

$$
H_{\mu \nu \lambda}= \pm \epsilon_{\mu \nu \lambda \sigma} \partial^{\sigma} \phi
$$

where $\mu, \nu, \ldots$ are indices for the transverse space $M^{4}$ and $\phi=\phi\left(x^{\mu}\right)$, while we shall use indices $a, b, \ldots$ for $M^{1,5}$.

Then other equations are solved by constant chiral spinors $\epsilon_{ \pm}$and

$$
g_{a b}=\eta_{a b}, \quad g_{\mu \nu}=\mathrm{e}^{-2 \phi} \delta_{\mu \nu}
$$

such that

$$
\begin{aligned}
& \delta \psi_{\mu}=\left(\nabla_{\mu}+\frac{1}{2} \Gamma_{\mu \nu} \partial^{\nu} \phi\right) \epsilon_{ \pm}=\partial_{\mu} \epsilon_{ \pm}=0 \\
& \delta \psi_{a}=\nabla_{a} \epsilon_{ \pm}=\partial_{a} \epsilon_{ \pm}=0
\end{aligned}
$$

and

$$
\delta \chi=F_{\mu \nu}^{ \pm} \Gamma^{\mu \nu} \epsilon_{ \pm}=-F_{\mu \nu}^{ \pm} \Gamma^{\mu \nu} \epsilon_{ \pm}=0
$$

where eq.(9) is achieved using the instanton configuration for the (anti)self-dual YM equation in $M^{4}$

$$
F_{\mu \nu}^{ \pm}= \pm \frac{1}{2} \epsilon_{\mu \nu}^{\rho \sigma} F_{\rho \sigma}^{ \pm}
$$

for an $\mathrm{SU}(2)$ subgroup of $E_{8} \times E_{8}$ or $\mathrm{SO}(32)$.

Solutions of eq.(10) are basic ingredients to build fivebrane solutions. For example, the instanton solutions lead to the Strominger's fivebrane solutions. In this case the basic fivebrane solution is called the "gauge" solution. Note that $\phi=\phi\left(r^{2}\right)$ now, i.e. no angular dependence, where $r^{2}=\sum\left(x^{\mu}\right)^{2}$ so that the transverse space has a rotational symmetry. With a finite instanton scale size $\lambda$, from eqs.(4)(6) we obtain

$$
\mathrm{e}^{-2 \phi}=\mathrm{e}^{-2 \phi_{0}}+8 \alpha^{\prime} \frac{\left(r^{2}+2 \lambda^{2}\right)}{\left(r^{2}+\lambda^{2}\right)^{2}}+\mathcal{O}\left(\alpha^{\prime 2}\right),
$$

where $\phi_{0}$ is the value of the dilaton at spatial infinity. Thus we have a fivebrane living in $M^{1,5}$ which is a point-like object in $M^{4}$.

Now we would like to call the reader's attention to the fact that any solution of eq.(10) in principle leads to a fivebrane solution, as long as the anomaly equation eq.(4) provides 
a nontrivial solution for the dilaton. In particular many lower dimensional solutions to the self-dual YM equation are known[10] so that in principle we can relate all these solitonic solutions to the heterotic fivebranes.

\section{Euclidean Sine-Gordon Case}

The (anti)self-dual YM equations have an interesting reduction to the two-dimensional solitonic system, namely the sine-Gordon equation. Here we shall attempt a new reduction of the (A)SDYM equation to the Euclidean sine-Gordon equation for the gauge group $\mathrm{SU}(2)$ and the Euclidean signature, then to solve the anomaly equation eq.(幽) for this solution. For the Euclidean signature we can introduce two sets of complex coordinates for convenience, although one can use the real coordinates, as

$$
z=x+i y, \bar{z}=x-i y, \quad w=u+i v, \bar{w}=u-i v
$$

where $(x, y, u, v)$ are the cartesian coordinates. In this coordinate system the SDYM equations will be written as

$$
F_{z \bar{z}}-F_{w \bar{w}}=0, \quad F_{z \bar{w}}=0, \quad F_{\bar{z} w}=0
$$

while the ASDYM equations are

$$
F_{z \bar{z}}+F_{w \bar{w}}=0, \quad F_{z w}=0, \quad F_{\overline{z w}}=0
$$

For the gauge group $\mathrm{SU}(2)$ with the generators $J_{ \pm}=\frac{1}{\sqrt{2}}\left(J_{1} \pm i J_{2}\right), \quad J_{3}$, which are in the adjoint representation such that $\left(J_{a}\right)_{b c}=-i \epsilon_{a b c}$, we can introduce an ansatz for the gauge fields as

$$
A_{z}=f_{1} J_{3}, A_{\bar{z}}=f_{2} J_{3}, A_{w}=g_{1} J_{+}+g_{3} J_{-}, A_{\bar{w}}=g_{2} J_{-}+g_{4} J_{+} .
$$

With such identifications the SDYM equations reduce to

$$
\begin{aligned}
f_{1} & =\partial_{z} \ln g_{2}=-\partial_{z} \ln g_{4} \\
f_{2} & =-\partial_{\bar{z}} \ln g_{1}=\partial_{\bar{z}} \ln g_{3}, \\
0 & =\partial_{z} f_{2}-\partial_{\bar{z}} f_{1}-g_{1} g_{2}+g_{3} g_{4}
\end{aligned}
$$

and the conditions that $\partial_{\bar{w}} f_{1}=\partial_{w} f_{2}=0, \partial_{w} g_{2}=\partial_{\bar{w}} g_{3}, \partial_{w} g_{4}=\partial_{\bar{w}} g_{1}$. The last conditions can be simply satisfied by requiring two Killing symmetries along $(u, v)$ directions that 
none of the fields depend on the $(u, v)$-coordinates. For the ASDYM equation we obtain more or less the same set of equations.

Now defining

$$
g_{1}=-g_{2}=e^{-\frac{i}{2} \psi}, g_{3}=-g_{4}=e^{\frac{i}{2} \psi},
$$

we obtain the Euclidean version of the sine-Gordon equation,

$$
\partial_{z} \partial_{\bar{z}} \psi-2 \sin \psi=\frac{1}{4}\left(\partial_{x}^{2}+\partial_{y}^{2}\right) \psi-2 \sin \psi=0
$$

The above is related, redefining $y=i t$, to the $\left(m^{2}=8\right)$ sine-Gordon equation

$$
\left(\partial_{t}^{2}-\partial_{x}^{2}\right) \varphi+\frac{m^{2}}{\lambda} \sin \lambda \varphi=0
$$

where the coupling constant $\lambda$ can be rescaled away since we are not interested in quantizing this system here.

In this background the anomaly equation eq.(雨 becomes up the first order of $\alpha^{\prime}$

$$
\left(\partial_{x}^{2}+\partial_{y}^{2}\right) \mathrm{e}^{-2 \phi}=2 \alpha^{\prime}\left[\sin \psi\left(\partial_{x}^{2}+\partial_{y}^{2}\right) \psi+\cos \psi\left(\left(\partial_{x} \psi\right)^{2}+\left(\partial_{y} \psi\right)^{2}\right)\right]
$$

Using the above sine-Gordon equation we can easily solve this equation to obtain a solution

$$
\mathrm{e}^{-2 \phi}=\mathrm{e}^{-2 \phi_{0}}+2 \alpha^{\prime}(1-\cos \psi)
$$

where $\psi$ satisfies the sine-Gordon equation and $\phi_{0}$ is the value of the dilaton $\phi$ at $x, y=$ $\pm \infty$.

Due to the Derrick's theorem[11] applied to the Euclidean sine-Gordon theory, there is no finite-action static solution for $\psi$. Nevertheless, we can have infinite-action static solutions, which do not generate any tunnelling effect. In fact we can easily find the following solution:

$$
\psi=4 Q \tan ^{-1}\left[\gamma \mathrm{e}^{\alpha x+\beta y}\right]
$$

where $\gamma$ is an arbitrary irrelevant constant so that we can set $\gamma=1$ without loss of generality, and $\alpha^{2}+\beta^{2}=8 . Q= \pm 1$ is the soliton charge. This solution is related to the soliton solutions of the sine-Gordon equation eq.(19),

$$
\varphi=4 Q \tan ^{-1}\left[\exp m \frac{x-c t}{\sqrt{1-c^{2}}}\right]
$$


identifying

$$
y=i t, \quad c=i \tilde{c}, \quad \alpha=\frac{m}{\sqrt{1+\tilde{c}^{2}}}, \quad \beta=\frac{m \tilde{c}}{\sqrt{1+\tilde{c}^{2}}}, \quad m=2 \sqrt{2} .
$$

It is straightforward to show that the corresponding action of the Euclidean sineGordon theory is indeed infinite for these solutions. However, this cannot be a reason to abandon these solutions for our purpose because this action is not an essential ingredient for fivebrane solutions. Note that the SDYM equation is not an equation of motion so that the action for any reduced system from the SDYM equation is not relevant to us. Due to the self-dual YM structure, the corresponding fivebrane solutions can still saturate the Bogomol'nyi bound of the action of the heterotic string theory. Strictly speaking, the fivebrane is not an instanton related to the tunnelling effect because we work on the $(1+9)$ dimensional spacetime. From this point of view, whether the action of the heterotic string is finite or not is not really a relevant issue to us. We are just interested in looking for some solitonic solutions.

Using eq.(22), now the dilaton eq.(21) becomes

$$
\mathrm{e}^{-2 \phi}=\mathrm{e}^{-2 \phi_{0}}+16 \alpha^{\prime} \frac{\mathrm{e}^{2(\alpha x+\beta y)}}{\left(\mathrm{e}^{2(\alpha x+\beta y)}+1\right)^{2}} .
$$

Note that this solution does not have any singularity and depends on the $x, y$-coordinates explicitly, not just on $x^{2}+y^{2}$. This dilaton solution does not care about the sign of the soliton charge $Q= \pm 1$, while the YM fields depend on the charge $Q= \pm 1$. We can also express the YM fields eq.(15) in terms of eq.(22) as follows:

$$
\begin{aligned}
A_{z} & =-Q(\beta+i \alpha) \frac{\mathrm{e}^{(\alpha x+\beta y)}}{\mathrm{e}^{2(\alpha x+\beta y)}+1} J_{3}, \\
A_{\bar{z}} & =Q(\beta-i \alpha) \frac{\mathrm{e}^{(\alpha x+\beta y)}}{\mathrm{e}^{2(\alpha x+\beta y)}+1} J_{3}, \\
A_{w} & =\frac{1-\mathrm{e}^{2(\alpha x+\beta y)}-i 2 Q \mathrm{e}^{(\alpha x+\beta y)}}{\mathrm{e}^{2(\alpha x+\beta y)}+1} J_{+}+\frac{1-\mathrm{e}^{2(\alpha x+\beta y)}+i 2 Q \mathrm{e}^{(\alpha x+\beta y)}}{\mathrm{e}^{2(\alpha x+\beta y)}+1} J_{-}, \\
A_{\bar{w}} & =\frac{1-\mathrm{e}^{2(\alpha x+\beta y)}-i 2 Q \mathrm{e}^{(\alpha x+\beta y)}}{\mathrm{e}^{2(\alpha x+\beta y)}+1} J_{-}+\frac{1-\mathrm{e}^{2(\alpha x+\beta y)}+i 2 Q \mathrm{e}^{(\alpha x+\beta y)}}{\mathrm{e}^{2(\alpha x+\beta y)}+1} J_{+} .
\end{aligned}
$$

The fact that there are all the four dimensional YM fields indicates that the solutions we have here are still fivebrane solutions.

Now let us count the zero modes. In the two-dimension parametrized by $(x, y)$ coordinates the soliton solutions eq.(22) generate four zero modes, which are two for the two 
translational symmetries of the $x, y$-directions, one for the $\left(\alpha^{2}+\beta^{2}=8\right)$ "scaling" symmetry and one for the $\mathrm{O}(2)$ rotational symmetry of $(\alpha x+\beta y)$. This last $\mathrm{O}(2)$ symmetry is due to the fact that the $\mathrm{O}(2)$ rotation of $(x, y)$ can be compensated by $\mathrm{O}(2)$ rotation of $(\alpha, \beta)$. Since the two Killing symmetries, $\left(\partial_{u}, \partial_{v}\right)$, generate four extra zero modes for the fivebrane, the fivebrane solution still has 120 bosonic zero modes, including 112 zero modes due to $E_{8} \rightarrow S U(2) \times E_{7}$, like in the "gauge" solution case. We expect that the fermionic zero modes counting is also similar to the "gauge" solution case.

\section{Discussion}

In general one could expect that the fivebranes could appear as particles, strings or membranes in the $(1+3)$ dimensional spacetime. In this letter we have presented an explicit construction of solitonic solutions in the two dimensional subspace of the transverse space $M^{4}$, reduced from the heterotic fivebrane solutions in ten-dimension. The fivebrane solution we have derived looks like a string in the $(1+3)$ dimensional space defined by $(x, y)$ plane and a $(1+1)$ dimensional subspace of $M^{1,5}$. Whether this solution should behave like a cosmic string is another issue because we have not mentioned anything about the symmetry breaking structure yet. But after we find out the detail of the compactification scheme, this should be an important issue to be addressed.

We also expect that the origin of the electric-magnetic duality in four-dimensional world is originated from the string-fivebrane duality in ten-dimension in such a way that the solitonic sector of the four-dimensional effective field theory might be coming from the fivebrane sector of the string theory.

From this point of view, further study of the properties of these solutions presented here should be important in future.

\section{Acknowledgements}

The author would like to thank M. Duff, D. Nanopoulos and E. Sezgin for conversations on related subjects. This work was supported in part by NSF grant PHY89-07887 and a World Laboratory Fellowship. 


\section{References}

[1] For recent reviews, see M.J. Duff and J.X. Lu, "A Duality between Strings and Fivebranes," Texas A\&M preprint, Class. Quant. Grav. 9 (1992) 1; C.G. Callan, J.A. Harvey and A. Strominger "Supersymmetric String Solitons ," Chicago preprint, EFI-9166 (1991); and references therein.

[2] M.J. Duff, Class. Quan. Grav. 5 (1988) 189;

M.J. Duff, in Superworld II, ed. by A. Zichichi (Plenum, New York, 1990).

[3] A. Strominger, Nucl. Phys. B343 (1990) 167.

[4] C. Montonen and D. Olive, Phys. Lett. 72B (1977) 117.

[5] J.A. Harvey and J. Liu, Phys. Lett. 268B (1991) 40.

[6] R. Khuri and H.S. La, Texas A\&M preprint, CTP-TAMU-95/91, -98/91 (1991).

[7] H.S. La, "Solitons reduced from Heterotic Fivebranes," CTP-TAMU 36/92 (1992).

[8] H.S. La, in preparation.

[9] C.G. Callan, J. Harvey and A. Strominger, Nucl. Phys. B359 (1991) 611.

[10] R.S. Ward, "Integrable Systems in Twistor Theory," in Twistors in Mathematics and Physics, (Cambridge Univ. Press, 1990).

[11] G.H. Derrick, J. Math. Phys. 5 (1964) 1252;

R. Hobart, Proc. Phys. Soc. 82 (1963) 201. 